\title{
Noninvasive Models for Predicting Liver Fibrosis in Individuals with Hepatitis D Virus/Hepatitis $B$ Virus Coinfection in the Brazilian Amazon Region
}

\author{
Mariana Pinheiro Alves Vasconcelos, ${ }^{1,2 \star}$ Deusilene Vieira DallÁcqua, ${ }^{1,3}$ Heiner Wedemeyer, ${ }^{4}$ Steven S. Witkin, ${ }^{5,6}$ \\ Maria Cássia Mendes-Corrêa, ${ }^{2,6}$ and Juan Miguel Villalobos-Salcedo ${ }^{1,3}$
}

${ }^{1}$ Research Center for Tropical Medicine of Rondônia, Porto Velho, Brazil; ${ }^{2}$ Department of Infectious Diseases, University of São Paulo, School of Medicine, Brazil; ${ }^{3}$ Oswaldo Cruz Institute of Rondônia, Porto Velho, Brazil; ${ }^{4}$ Essen University Hospital, Essen, Germany; ${ }^{5}$ Weill Cornell Medicine, New York, New York; 'Institute of Tropical Medicine, University of São Paulo, São Paulo, Brazil

\begin{abstract}
Hepatitis D virus (HDV) genotype III is endemic in the western Amazon basin and is considered to cause the most severe form of chronic viral hepatitis. Recently, noninvasive fibrosis scores to determine the stage of liver fibrosis have been evaluated in individuals positive for HDV genotype I, but their utility in HDV genotype III-positive patients is unknown. In this retrospective study conducted in an outpatient viral hepatitis referral clinic in the Brazilian Amazon region, the aspartate aminotransferase (AST) to Aspartate aminotransferase to Platelet Ratio Index (APRI) and Fibrosis Index for Liver Fibrosis (FIB-4) values were calculated and compared with histological fibrosis stages. Among the 50 patients analyzed, the median age at liver biopsy was 35.6 years, 66\% were male, and all had compensated liver disease. Histological staging revealed fibrosis stages $0,1,2,3$, and 4 in four (8\%), eight (16\%), $11(22), 11(22 \%)$, and $16(32 \%)$ patients, respectively. The area under the receiver operating curve (AUROC) of AST-to-alanine aminotransferase (ALT) ratio, APRI, and FIB-4 for detection of significant fibrosis $(\mathrm{F} \geq 2)$ was $0.550(P=0.601), 0.853(P<0.001)$, and $0.853(P<$ $0.0001)$, respectively. Lower AUROC values were obtained for cirrhosis: the AST-to-ALT ratio was $0.640(P=0.114)$, APRI was $0.671(P=0.053)$, and FIB-4 was $0.701(P=0.023)$. The optimal cutoff value for significant fibrosis for APRI was 0.708 (sensitivity $84 \%$ and specificity $92 \%$ ) and for FIB-4 was 1.36 (sensitivity $76 \%$ and specificity $92 \%$ ). Aspartate aminotransferase to Platelet Ratio Index and FIB-4 were less useful to predict cirrhosis. In contrast to recent reports from Europe and North America, both APRI and FIB-4 may identify significant fibrosis in HDV-III-infected patients from northwestern Brazil.
\end{abstract}

\section{INTRODUCTION}

The WHO estimated that in 2015, 257 million people were living with chronic hepatitis B virus (HBV) infection. Also according to the $\mathrm{WHO}$, at least $5 \%$ of people with chronic HBV infection are coinfected with hepatitis $D$ virus (HDV), resulting in a total of 15-20 million persons infected with HDV worldwide. ${ }^{1}$ Hepatitis D virus is a capsid-deficient RNA virus which uses the HBV surface antigen (HBsAg) to form infectious particles. ${ }^{2,3}$

The western Amazon basin, including Brazil, Peru, Ecuador, Venezuela, and Colombia, is considered an endemic area for HBV and HDV infection. ${ }^{4}$ In Brazil, this area corresponds to the states of Acre, Amazonas, Rondônia, and Roraima, with a high prevalence in the indigenous population. ${ }^{5}$

Chronic HDV is the most severe form of chronic viral hepatitis with rapid progression to cirrhosis and with limited treatment options. Genotype III HDV is endemic in the western Amazon basin and is associated with rapid progression of liver disease. Pegylated-interferon alpha is currently the only therapy licensed for the treatment of chronic HDV, but only $25 \%$ of patients have a sustained antiviral response after 1 year of treatment. ${ }^{6-8}$ The gold reference standard for determining hepatic histology is liver biopsy. However, liver biopsy is an invasive procedure that has been associated with complications. In addition, chronic HDV infection has been described mainly in areas with limited resources, which often makes liver biopsy or transient elastography inaccessible. Consequently, there has been considerable interest in identification of noninvasive

\footnotetext{
*Address correspondence to Mariana Pinheiro Alves Vasconcelos, Centro de Pesquisa em Medicina Tropical de Rondônia, Avenida Guaporé, 215, Porto Velho, Rondônia 78918-791, Brazil. E-mail:
} marianapvasconcelos@hotmail.com markers to accurately evaluate the extent of hepatic involvement among chronic HDV-infected patients.

Noninvasive fibrosis assessment using transient elastography acoustic radiation force impulse imaging or shear wave elastography has been applied in HDV infection, but high costs limit its application in settings where HDV is highly prevalent. ${ }^{9}$ Studies assessing the accuracy of scores derived from standard blood tests, e.g., aspartate transaminase (AST)to-alanine transaminase (ALT) ratio, aspartate aminotransferaseto-platelet ratio (APRI), and fibrosis index based on four factors (FIB-4) in HDV are limited and predominantly involve individuals infected with HDV genotype I. $^{10}$ The aim of this study was to evaluate the accuracy of fibrosis stratification by AST-to-ALT ratio, $\mathrm{APRI}$, and FIB-4 compared with liver biopsy in patients with HDV genotype III infections.

\section{METHODS}

Study population. This retrospective cross-sectional study was performed using a cohort of chronic HDV patients followed at a dedicated chronic viral hepatitis ambulatory setting Centro de Pesquisa em Medicina Tropical (CEPEM), in Rondônia state, Brazilian Amazon region. Eligible patients included all those with chronic HDV infection characterized by positive anti-HDV antibody or HDV-RNA in serum and who were evaluated and followed at CEPEM between 1996 and 2015. The analysis included consecutive subjects with chronic HDV infection who underwent a liver biopsy and had concurrent laboratory assessments (blood test results within 6 months of biopsy) that included routine liver tests and blood counts.

Patients were not included in the study if 1) laboratory tests revealed ALT or AST levels more than eight times above the upper limit of normal (ULN) at the time of liver biopsy and 
laboratory assessments, 2) they presented with any other concomitant liver disease, 3) their liver biopsies were not considered representative (less than 10 portal spaces in the histopathological assessment) by the on-site pathologist, 4) they received any treatment for HBV or HDV before the liver biopsy was performed, and 5) they tested positive for HIV or hepatitis $C$ virus by commercial diagnostic tests.

Liver biopsy and histology. Liver biopsies. Transcutaneous liver biopsies were guided by ultrasound and performed only in clinically biopsy-indicated patients. Liver fragments were fixed, embedded in paraffin, and stained with hematoxylin-eosin, Masson's trichrome, and reticulin (for collagen). Liver biopsy specimens were evaluated and scored by experienced hepatopathologists.

Only biopsies with adequate tissue were scored, which was defined as a minimum of 10 portal tracts visualized. Histological findings were classified according to the Meta-analysis of Histological Data in Viral Hepatitis (METAVIR) classification scoring system (F0: no fibrosis; F1: portal fibrosis without septa; F2: portal fibrosis with rare septa; F3: numerous septa without cirrhosis; and F4: cirrhosis). ${ }^{11}$ For our study, significant fibrosis was defined as fibrosis stage $\geq 2$ (F2, F3, and F4), advanced fibrosis was defined as fibrosis stage $\geq 3$ (F3 and F4), and cirrhosis was defined as fibrosis stage F4.

Data collection and laboratory tests. Medical records were retrospectively reviewed to obtain clinical and demographic characteristics. Laboratory data were also reviewed. Variables described were age, gender, and mode of HBV/HDV transmission. Hepatitis B virus serological testing was performed according to standard procedures and using licensed commercial assays, including $\mathrm{HBsAg}$, total anti-HBc, anti$\mathrm{HBs}, \mathrm{HBeAg}$, and anti-HBe. Hepatitis D virus coinfection was defined by the presence of positive anti-HDV antibodies by ELISA using commercially available kits from DiaSorin. ${ }^{12}$ Hepatitis D virus infection was confirmed by polymerase chain reaction ( $P C R$ ) in $R N A$, and the HDV genotype was determined by sequencing and phylogenetic analysis, as previously reported. ${ }^{13,14}$ Hepatitis $\mathrm{C}$ and HIV serological tests were also performed on all potential study subjects.

Biochemical markers of liver fibrosis. For all included patients, the AST-to-ALT ratio, APRI [(AST/ULN value:45IU/L)/ platelet counts (109/L)] x 100, and FIB-4 [age (year) $\times$ AST(UI/ $\mathrm{L}) /$ platelet counts $(109 / \mathrm{L}) \times \sqrt{ } \mathrm{ALT}(\mathrm{UI} / \mathrm{L})]$ were calculated. ${ }^{15-17}$ The ULN for ALT and AST was considered as follows: $45 \mathrm{IU} / \mathrm{L}$ and $38 \mathrm{IU} / \mathrm{L}$, respectively.

Ethics. The study was conducted after obtaining ethical approval from the Center for Research in Tropical Medicine in Rondônia Ethical Committee (CEP/CEPEM 055063/2015). All patients who returned for follow-up signed the informed consent form. For patients no longer available for follow-up, the Ethics Committee granted a waiver of the need for a signed consent form.

Statistical analysis. Statistical analysis was performed by IBM $^{\circledR}$ SPSS $^{\circledR}$ Statistics version 24.0 (SPSS, Chicago, IL). The diagnostic performance of the AST-to-ALT ratio, APRI, and FIB-4 for detecting significant fibrosis (F2, F3, and F4), advanced fibrosis (F3 and F4), and cirrhosis (F4) was assessed with receiver operating characteristic curves the and associated area under the curve. Sensitivity, specificity, positive predictive value (PPV), and negative predictive value (NPV) were also calculated for significant fibrosis, advanced fibrosis, and cirrhosis.

\section{RESULTS}

Among a total of 205 eligible patients, 85 had undergone liver biopsy and only 50 were included in this study. Among the 35 excluded patients, 11 presented with nonrepresentative liver biopsies (less than 10 portal spaces in the histopathological assessment), 22 lacked laboratory data, and two had transaminase levels more than eight times above the ULN.

Patient characteristics. The characteristics of included patients are shown in Table 1. The median age was 35.6 12.6 years, 33 (66\%) were male, 17 (34\%) were female, and nine $(18 \%)$ were of indigenous ethnicity. Intrafamilial contact was the main risk factor observed (50\%).

All patients were $\mathrm{HBsAg}$ positive, anti-HBc lgG positive, and anti-HDV positive. Among all patients, only (8\%) were HBeAg positive. Only 24 (44\%) had their diagnosis confirmed by HDV RNA analysis and genotype identification, two (8.3\%) of which were genotype I and 22 (91.7\%) were genotype III; all the other patients had only serological diagnosis of HDV.

Biomarker performances. The AST-to-ALT ratio, APRI, and FIB-4 scores observed in relation to liver fibrosis stage following liver biopsy are shown in Table 2. The area under the receiver operating curve (AUROC) for detecting significant fibrosis was 0.550 for the AST-to-ALT ratio $(P=0.601), 0.853$ for APRI $(P<0.001)$, and 0.853 for FIB-4 $(P<0.001)$ (Figure 1). For advanced fibrosis, the AUROC was 0.517 for the AST-to-ALT ratio $(P=0.838), 0.839$ for $\mathrm{APRI}(P<0.001)$, and 0.781 for FIB-4 $(P=0.001)$ (Figure 2). Last, for cirrhosis, the AUROC was 0.640 for the AST-to-ALT ratio $(P=0.114), 0.671$ for APRI $(P=0.053)$, and 0.701 for FIB-4 $(P=0.023)$ (Figure 3$)$.

Optimal cutoffs were determined which maximized the PPV (Table 3). For significant fibrosis, the cutoff for APRI was 0.708 (84\% sensitivity, 92\% specificity, 97\% PPV, and 65\% NPV. The cutoff for FIB-4 was 1.36 (76\% sensitivity, 92\% specificity, 97\% PPV, and 55\% NPV). For advanced fibrosis, the APRI cutoff was 0.933 (89\% sensitivity, $74 \%$ specificity, $80 \%$ PPV, and $85 \%$ NPV). For FIB-4, the cutoff was $1.36(81 \%$ sensitivity, $65 \%$ specificity, $73 \%$ PPV, and $75 \%$ NPV). Finally,

TABLE 1

Characteristics of patients in the cohort

\begin{tabular}{lc}
\hline \multicolumn{1}{c}{ Patients characteristic } & $N=50$ \\
\hline Age (years), median & 35.6 \\
Male, $n$ (\%) & $33(66)$ \\
Ethnicity, $n$ (\%) & \\
Caucasian & $3(6)$ \\
Indigenous & $9(18)$ \\
Mixed & $31(62)$ \\
Black & $3(6)$ \\
Missed information & $4(8)$ \\
From Amazon region, $n(\%)$ & $40(80)$ \\
Interfamily contact, $n$ (\%) & \\
Hepatitis B virus & $25(50)$ \\
Hepatitis D virus & $8(16)$ \\
Genotype, $n$ (\%) & \\
Genotype I & $2(4)$ \\
Genotype III & $22(44)$ \\
Missed information & $26(52)$ \\
Fibrosis stage (METAVIR), $n(\%)$ & \\
F0 & $4(8)$ \\
F1 & $8(16)$ \\
F2 & $11(22)$ \\
F3 & $11(22)$ \\
F4 & $16(32)$ \\
HBeAg positive & $4(8)$ \\
\hline
\end{tabular}


TABLE 2

Laboratory parameters and mean scores of the three biomarkers in increasing stages of liver fibrosis

\begin{tabular}{|c|c|c|c|c|c|c|}
\hline & Total $(n=50)$ & FO $(n=4)$ & F1 $(n=8)$ & F2 $(n=11)$ & F3 $(n=11)$ & F4 $(n=16)$ \\
\hline \multicolumn{7}{|c|}{ Platelets (109/L) } \\
\hline Mean \pm SD & $177 \pm 95.4$ & $189 \pm 20.8$ & $286 \pm 118.1$ & $189 \pm 95.7$ & $123 \pm 49.9$ & $147 \pm 76.2$ \\
\hline Range & $50-490$ & $178-221$ & $164-490$ & $98-380$ & 50-204 & $50-320$ \\
\hline \multicolumn{7}{|l|}{ ALT (IU/L) } \\
\hline Mean \pm SD & $80.5 \pm 58.3$ & $90.5 \pm 93.3$ & $37.2 \pm 27.1$ & $61.5 \pm 36.5$ & $125.1 \pm 54.4$ & $81.8 \pm 58.4$ \\
\hline Range & $11-257$ & $35-230$ & $11-98$ & $12-130$ & $36-192$ & $26-257$ \\
\hline \multicolumn{7}{|l|}{ AST (IU/L) } \\
\hline Mean \pm SD & $71.3 \pm 54.7$ & $78.2 \pm 90.5$ & $30.5 \pm 15.1$ & $55.2 \pm 34.5$ & $101.0 \pm 68.4$ & $80.7 \pm 47.9$ \\
\hline Range & 13-297 & $30-214$ & 13-58 & $15-120$ & $45-297$ & $30-214$ \\
\hline \multicolumn{7}{|l|}{ AST/ALT } \\
\hline Mean \pm SD & $0.96 \pm 0.4$ & $0.81 \pm 0.2$ & $0.91 \pm 0.2$ & $0.94 \pm 0.2$ & $0.91 \pm 0.5$ & $1.08 \pm 0.5$ \\
\hline Range & $0.42-2.45$ & $0.57-0.93$ & $0.59-1.18$ & $0.66-1.36$ & $0.42-2.17$ & $0.50-2.45$ \\
\hline \multicolumn{7}{|c|}{ Aspartate aminotransferase to Platelet Ratio Index } \\
\hline Mean \pm SD & $1.57 \pm 1.8$ & $1.16 \pm 1.4$ & $0.34 \pm 0.2$ & $1.00 \pm 0.7$ & $2.61 \pm 2.6$ & $1.95 \pm 1.7$ \\
\hline Range & $0.08-10.03$ & $0.37-3.34$ & $0.08-0.70$ & $0.11-2.36$ & $1.19-10.03$ & $0.44-7.82$ \\
\hline \multicolumn{7}{|l|}{ FIB-4 } \\
\hline Mean \pm SD & $2.25 \pm 2.3$ & $1.18 \pm 0.4$ & $0.63 \pm 0.4$ & $1.82 \pm 1.3$ & $3.57 \pm 3.9$ & $2.71 \pm 1.5$ \\
\hline Range & $0.16-14.15$ & $0.64-1.51$ & $0.16-1.23$ & $0.23-3.94$ & $0.29-14.15$ & $0.47-6.16$ \\
\hline
\end{tabular}

for cirrhosis, the cutoff for FIB-4 was 2.26 (62\% sensitivity, $79 \%$ specificity, 59\% PPV, and 82\% NPV).

\section{DISCUSSION}

Hepatitis D virus diagnosis and assessment in resourcepoor endemic settings present major challenges. Identifying infected individuals with significant liver disease who require urgent treatment is hampered by the high cost and availability of biopsy as well as other fibrosis tests such as liver-specific serum markers and liver elastography. The present study was conducted to evaluate the accuracy of fibrosis stratification by the AST-to-ALT ratio, APRI, and FIB-4 as compared with liver biopsy in patients with chronic HDV infection. We demonstrate that the APRI and FIB-4 scores, derived from commonly performed blood tests, identify patients with significant and advanced disease with high PPV and NPV. Our data also confirm, as observed previously, that AST/ALT has a low sensitivity in establishing fibrosis staging among chronic HDV-infected patients. ${ }^{9}$

Aspartate aminotransferase to Platelet Ratio Index, in particular, was very efficient in identifying F3 patients, with high PPV

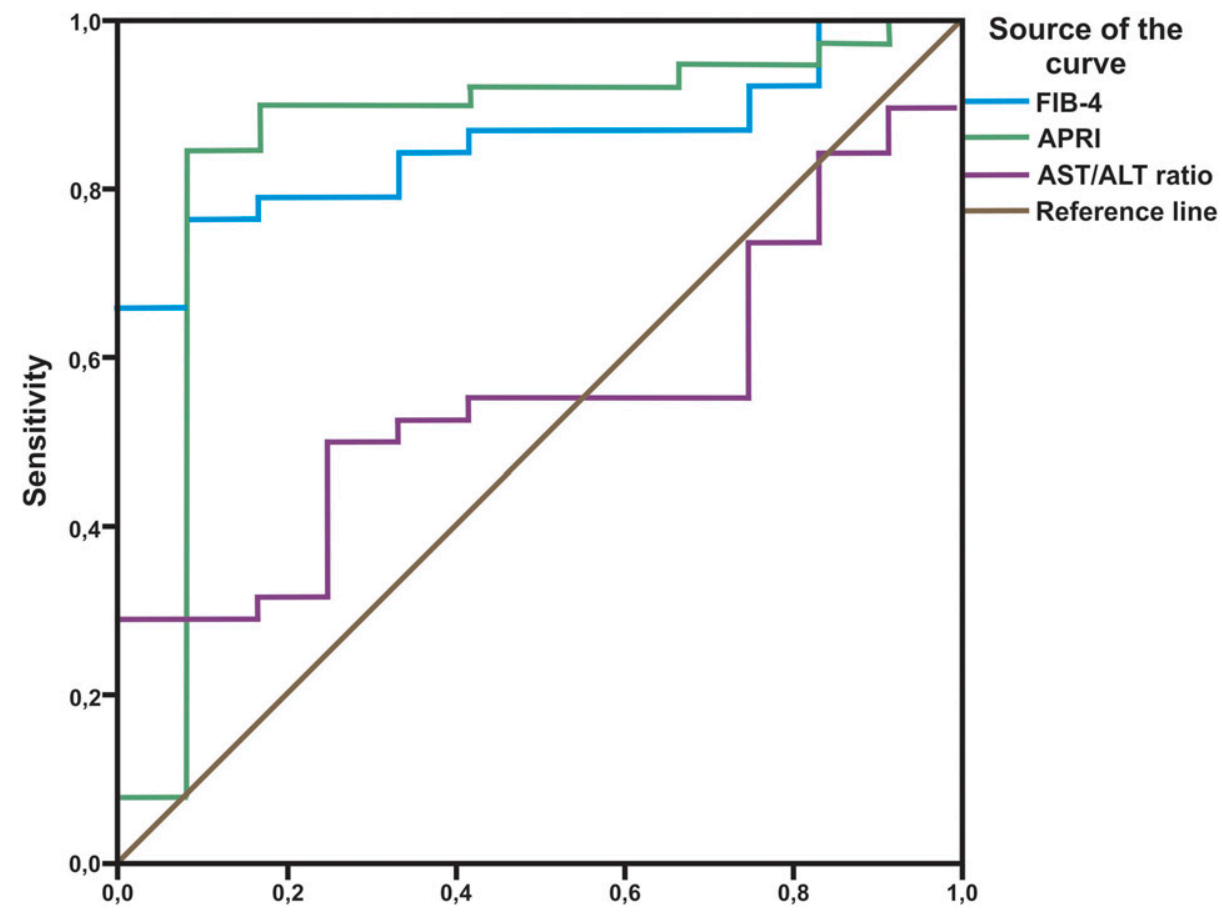

FIGURE 1. Receiver operating curves for aspartate aminotransferase (AST)/alanine aminotransferase (ALT), Aspartate aminotransferase to Platelet Ratio Index (APRI), and FIB-4 for detection of significant fibrosis (F2, F3, and F4). This figure appears in color at www.ajtmh.org. 


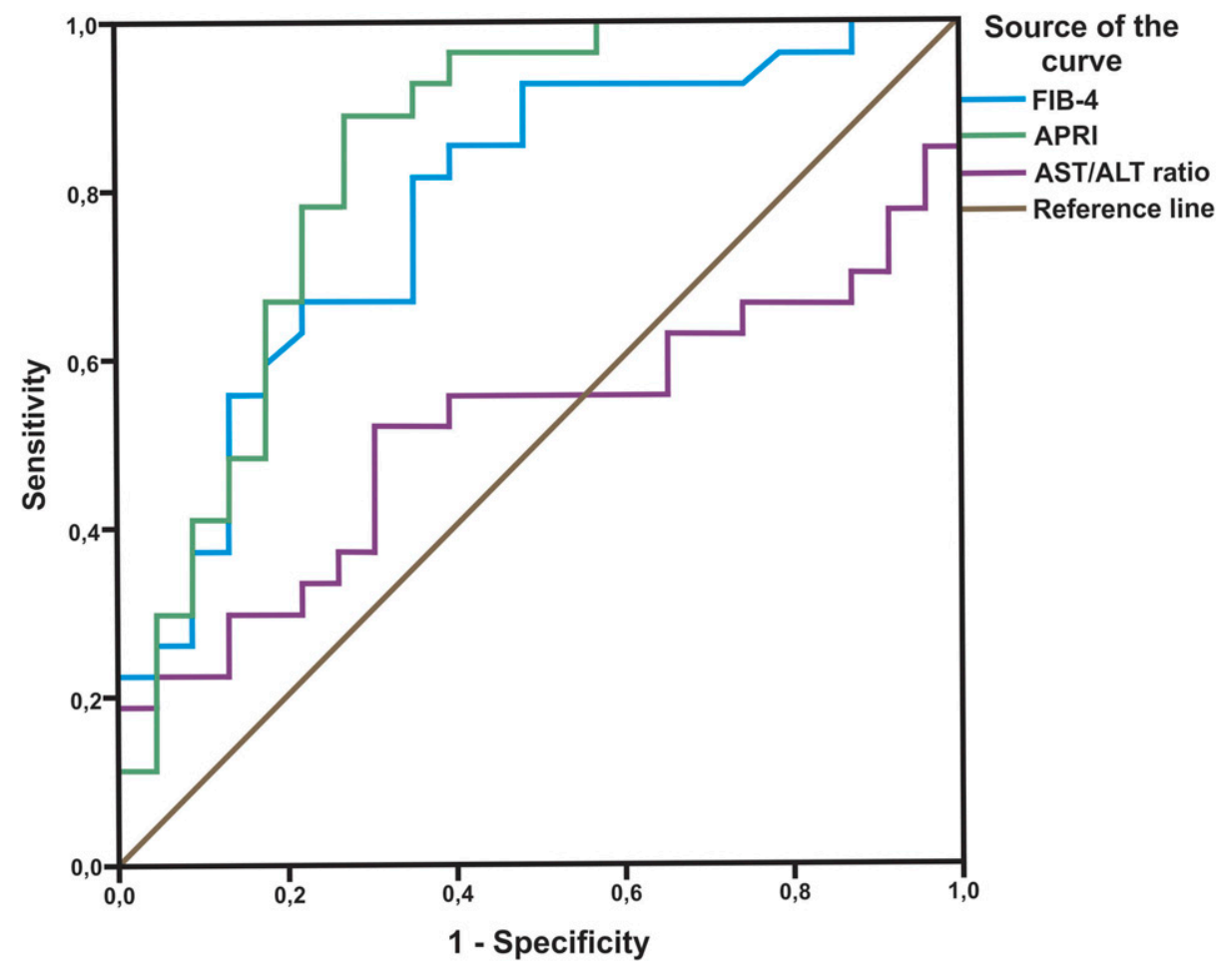

FIGURE 2. Receiver operating curves for aspartate aminotransferase (AST)/alanine aminotransferase (ALT), Aspartate aminotransferase to Platelet Ratio Index (APRI), and FIB-4 for detection of severe fibrosis (F3 and F4). This figure appears in color at www.ajtmh.org.

and NPV. Identification of such individuals by noninvasive methods is certainly more difficult than the documentation of cirrhotic patients. Cirrhotic patients can be identified by a number of clinical and laboratory characteristics that are not usually present in F3 patients. However, similar to those with cirrhosis, F3-positive individuals need continuous follow-up and treatment. These findings are in contrast to recent reports from Europe and North America which evaluated the utility of APRI and

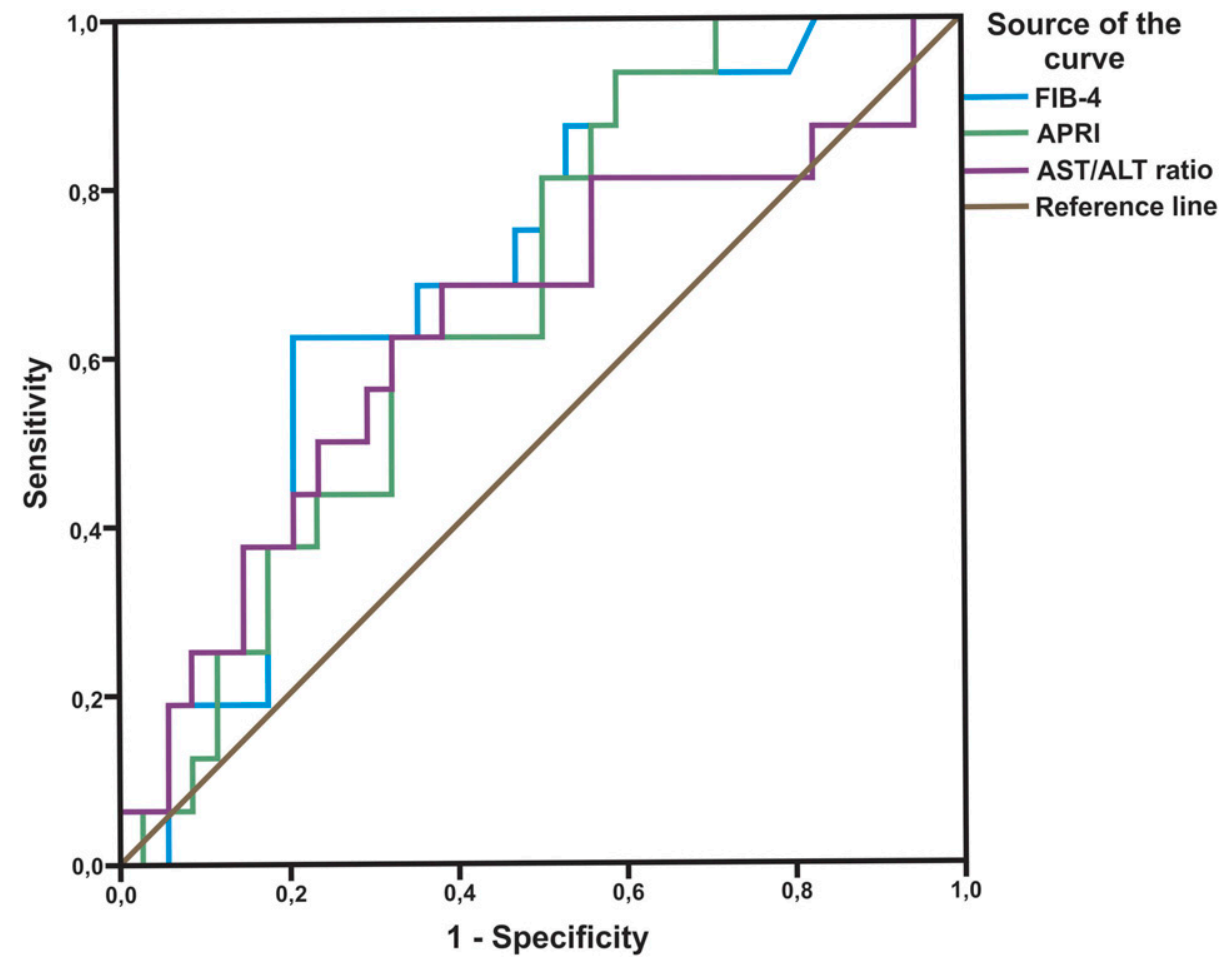

FIGURE 3. Receiver operating curves for aspartate aminotransferase (AST)/alanine aminotransferase (ALT), Aspartate aminotransferase to Platelet Ratio Index (APRI), and FIB-4 for detection of cirrhosis (F4). This figure appears in color at www.ajtmh.org. 
TABLE 3

Diagnostic performance of noninvasive methods for significant fibrosis, advanced fibrosis, and cirrhosis

\begin{tabular}{|c|c|c|c|c|c|c|c|c|c|}
\hline & Score & Area & $P$-value & Cutoff $^{*}$ & Sensitivity & Specificity & Positive predictive value & Negative predictive value & Accuracy \\
\hline \multirow[t]{3}{*}{ Significant fibrosis $(F \geq 2)$} & AST/ALT & 0.550 & 0.601 & 1.183 & $34 \%$ & $92 \%$ & $93 \%$ & $31 \%$ & 48.0 \\
\hline & APRI & 0.853 & $<0.001$ & 0.708 & $84 \%$ & $92 \%$ & $97 \%$ & $65 \%$ & 86.0 \\
\hline & FIB-4 & 0.853 & $<0.001$ & 1.360 & $76 \%$ & $92 \%$ & $97 \%$ & $55 \%$ & 80.0 \\
\hline \multirow[t]{3}{*}{ Advanced fibrosis $(F \geq 3)$} & AST/ALT & 0.517 & 0.838 & 0.960 & $52 \%$ & $65 \%$ & $64 \%$ & $54 \%$ & 58.0 \\
\hline & APRI & 0.839 & $<0.001$ & 0.933 & $89 \%$ & $74 \%$ & $80 \%$ & $85 \%$ & 82.0 \\
\hline & FIB-4 & 0.781 & 0.001 & 1.360 & $81 \%$ & $65 \%$ & $73 \%$ & $75 \%$ & 74.0 \\
\hline \multirow[t]{3}{*}{ Cirrhosis $(F=4)$} & AST/ALT & 0.640 & 0.114 & 0.960 & $62 \%$ & $65 \%$ & $45 \%$ & $79 \%$ & 64.0 \\
\hline & APRI & 0.671 & 0.053 & 0.656 & $94 \%$ & $41 \%$ & $43 \%$ & $93 \%$ & 58.0 \\
\hline & FIB-4 & 0.701 & 0.023 & 2.265 & $62 \%$ & $79 \%$ & $59 \%$ & $82 \%$ & 74.0 \\
\hline
\end{tabular}

${ }^{\star}$ The optimal cutoff point was determined by the point at which the true-positive rate is optimized and the false-positive rate is minimized.

FIB-4 in HDV genotype I-positive individuals. In these studies, the serological tests did not specifically identify patients with significant and advanced fibrosis. ${ }^{9,10}$ Perhaps, the utility of $\mathrm{APRI}$ and FIB-4 ratio analysis, as described in these studies, cannot be extrapolated to other HDV genotypes that typically produce less severe disease. Genotype III HDV infections are known to produce the most aggressive inflammation and unique patterns of liver function test derangement. ${ }^{18}$ Viral genotyping was available for 24 of the 50 patients in our study, and $91.7 \%$ of those tested were positive for HDV genotype III. Because all our subjects were from the same geographical area, it is reasonable to assume that genotype III was also most prevalent among those whose genotyping was not available.

Despite the relatively low median age (35.6 \pm 12.6 years) of our patients, $54 \%$ already had advanced fibrosis $(F \geq 3)$ or cirrhosis (F4). We believe that this observation supports our conclusion that HDV 3 is the most prevalent genotype involved in the development of aggressive liver disease.

Our study also indicates that the performance of FIB-4 is superior to that of APRI in detecting liver cirrhosis. This observation is consistent with the data from other studies investigating score performances of $\mathrm{APRI}$ and FIB-4 among hepatitis B-infected patients. Bonnard et al. compared noninvasive methods to diagnose significant fibrosis in HBVpositive patients in Burkina Faso and described the AUROC for significant fibrosis ( $F \geq 2$ ) as 0.61 for APRI and 0.71 for FIB4. ${ }^{19}$ Also, the AUROC for cirrhosis was 0.50 for APRI and 0.74 for FIB-4. Similarly, a recent study by Zhang et al. ${ }^{20}$ demonstrated that the AUROC for FIB-4 to predict significant fibrosis, advanced fibrosis, and cirrhosis was $0.646,0.670$, and 0.715 , respectively. The AUROC for APRI for prediction of significant fibrosis, advanced fibrosis, and cirrhosis, was $0.656,0.653$, and 0.639 , respectively. ${ }^{20}$

Although our results appear to be similar to the those of HBV studies, further research is nevertheless needed to determine the optimal cutoff values to diagnose cirrhosis and the clinical utility of these noninvasive methods in HDV patients. Recently, Takjar et al. analyzed 62 patients with chronic HDV hepatitis and were unable to determine that FIB-4 was of value in identifying cirrhotic patients. However, in their study, only $16 \%$ of subjects had stage 4 fibrosis as compared with $32 \%$ described in our investigation. Perhaps, differences in the proportion of HDV stage 4 individuals in the two studies may have contributed to the variation in the FIB-4 analysis.

Limitations of our study must be recognized. A main limitation of our study arises from intrinsic characteristics of its retrospective design. Registration bias is an important component of this limitation because data were not collected through a rigid protocol developed for the survey. As a consequence, among the 50 included patients, only 24 (44\%) underwent diagnostic confirmation with HDV RNA and HDV genotype analysis and none had HBV genotyping performed. Unfortunately, most of the patients in the Amazon region have a poor follow-up history and, in addition, relevant laboratory tests are sometimes not available. Nevertheless, we believe that our analysis of the available cohort provides informative data to encourage further investigations on larger more divergent populations.

Another limitation of our study is that 35 of the 85 eligible subjects were excluded from the analysis. Eleven were excluded because of nonrepresentative liver biopsies. In general, the most likely reason for underrepresentation of liver tissue is fragmentation of the core biopsies. This tends to occur more commonly in samples from patients with stage 4 fibrotic disease. Therefore, it is possible that the proportion of cirrhotic patients in our study in fact may have been larger than the $32 \%$ that we reported. The inability to evaluate these subjects could have impacted the performance of APRI and FIB-4.

Access to rapid staging of liver disease is key to clinical management of HDV infection. The clinical application of noninvasive fibrosis tests to individuals infected with HDV genotype III, the most aggressive form of viral hepatitis that is endemic in the Amazon region and in other resource-limited settings, deserves further study. The results reported here will hopefully contribute to enhanced interest in development of larger studies.

Received September 15, 2019. Accepted for publication April 9, 2020. Published online May 18, 2020.

Disclosure: HW reports personal fees and non-financial support from Roche Diagnostics, personal fees and non-financial support from Abbott, personal fees and non-financial support from Eiger, non-financial support from MYR GmbH, outside the submitted work; and an honoraria for consulting and research support by companies developing diagnostic tools and antiviral therapies for viral hepatitis $B$ and $\mathrm{C}$.

Authors' addresses: Mariana Pinheiro Alves Vasconcelos, Deusilene Vieira DallÁcqua, and Juan Miguel Villalobos-Salcedo, Centro de Pesquisa em Medicina Tropical de Rondônia, Porto Velho, Brazil, E-mails: vasconcelosmpa@gmail.com, deusylenebio@hotmail.com, and juanitto2001@yahoo.com.br. Heiner Wedemeyer, Essen University Hospital, Essen, Germany, E-mail: heiner.wedemeyer@ukessen.de. Steven S. Witkin, Weill Cornell Medicine, New York, NJ, E-mail: switkin@med.cornell.edu. Maria Cássia Mendes-Corrêa, Department of Infectious Diseases, University of São Paulo, School of Medicine, Brazil, E-mail: cassiamc@uol.com.br. 


\section{REFERENCES}

1. WHO, 2017. Global Hepatitis Report 2017. Available at: https:// apps.who.int/iris/handle/10665/255016. Accessed August 15, 2018.

2. Rizzetto M, Canese MG, Arico S, Crivelli O, Trepo C, Bonino F, Verme $\mathrm{G}, 1977$. Immunofluorescence detection of new antigen-antibody system (delta/anti-delta) associated to hepatitis B virus in liver and in serum of HBsAg carriers. Gut 18: 997-1003.

3. Taylor JM, 2006. Structure and replication of hepatitis delta virus RNA. Curr Top Microbiol Immunol 307: 1-23.

4. Crispim MA, Fraiji NA, Campello SC, Schriefer NA, Stefani MM, Kiesslich D, 2014. Molecular epidemiology of hepatitis B and hepatitis delta viruses circulating in the Western Amazon region, North Brazil. BMC Infect Dis 14: 94.

5. Fonseca JC, Simonetti SR, Schatzmayr HG, Castejon MJ, Cesario AL, Simonetti JP, 1988. Prevalence of infection with hepatitis delta virus (HDV) among carriers of hepatitis B surface antigen in Amazonas State, Brazil. Trans R Soc Trop Med Hyg 82: $469-471$.

6. Rizzetto M, Alavian SM, 2013. Hepatitis delta: the rediscovery. Clin Liver Dis 17: 475-487.

7. Heidrich $B$ et al., 2014. Late HDV RNA relapse after peginterferon alpha-based therapy of chronic hepatitis delta. Hepatology 60: 87-97.

8. Yurdaydin $\mathrm{C}$, Bozkaya $\mathrm{H}$, Karaaslan $\mathrm{H}$, Onder FO, Erkan OE, Yalcin K, Degertekin H, Bozdayi AM, Uzunalimoglu O, 2007. A pilot study of 2 years of interferon treatment in patients with chronic delta hepatitis. J Viral Hepat 14: 812-816.

9. Lutterkort GL et al., 2017. Non-invasive fibrosis score for hepatitis delta. Liver Int 37: 196-204.

10. Takyar V, Surana P, Kleiner DE, Wilkins K, Hoofnagle JH, Liang TJ, Heller T, Koh C, 2017. Noninvasive markers for staging fibrosis in chronic delta hepatitis. Aliment Pharmacol Ther 45: 127-138.

11. Bedossa P, Poynard T, 1996. An algorithm for the grading of activity in chronic hepatitis C. The METAVIR cooperative study group. Hepatology 24: 289-293.
12. Chow SK, Atienza EE, Cook L, Prince H, Slev $P$, Lape-Nixon $M$, Jerome KR, 2016. Comparison of enzyme immunoassays for detection of antibodies to hepatitis D virus in serum. Clin Vaccin Immunol 23: 732-734.

13. Botelho-Souza LF, dos Santos Ade O, Borzacov LM, Honda ER, Villalobos-Salcedo JM, Vieira DS, 2014. Development of a reverse transcription quantitative real-time PCR-based system for rapid detection and quantitation of hepatitis delta virus in the western Amazon region of Brazil. J Virol Methods 197: 19-24.

14. Botelho-Souza LF, Souza Vieira D, de Oliveira dos Santos A, Cunha Pereira AV, Villalobos-Salcedo JM, 2015. Characterization of the genotypic profile of hepatitis delta virus: isolation of HDV genotype- 1 in the western Amazon region of Brazil. Intervirology 58: 166-171.

15. Sheth SG, Flamm SL, Gordon FD, Chopra S, 1998. AST/ALT ratio predicts cirrhosis in patients with chronic hepatitis $C$ virus infection. Am J Gastroenterol 93: 44-48.

16. Wai CT, Greenson JK, Fontana RJ, Kalbfleisch JD, Marrero JA, Conjeevaram HS, Lok AS, 2003. A simple noninvasive index can predict both significant fibrosis and cirrhosis in patients with chronic hepatitis C. Hepatology 38: 518-526.

17. Sterling RK et al.; APRICOT Clinical Investigators, 2006. Development of a simple noninvasive index to predict significant fibrosis in patients with HIV/HCV coinfection. Hepatology 43: 1317-1325.

18. Casey JL, Niro GA, Engle RE, Vega A, Gomez H, McCarthy M, Watts DM, Hyams KC, Gerin JL, 1996. Hepatitis B virus (HBV)/ hepatitis $D$ virus (HDV) coinfection in outbreaks of acute hepatitis in the Peruvian Amazon basin: the roles of HDV genotype III and HBV genotype F. J Infect Dis 174: 920-926.

19. Bonnard $P$ et al., 2010. Comparison of elastography, serum marker scores, and histology for the assessment of liver fibrosis in hepatitis B virus (HBV)-infected patients in Burkina Faso. Am J Trop Med Hyg 82: 454-458.

20. Zhang Z, Wang G, Kang K, Wu G, Wang P, 2016. The diagnostic accuracy and clinical utility of three noninvasive models for predicting liver fibrosis in patients with HBV infection. PLOS One 11: e0152757. 